\title{
Selecting Sensor for On Load Tap Changer Contacts Degree of Wear Diagnostics
}

\author{
A. CiChON ${ }^{a, *}$, S. BORUCKI ${ }^{a}$ AND P. BerGer ${ }^{b}$ \\ ${ }^{a}$ Faculty of Electrical Engineering, Automatic Control and Computer Science, Opole University of Technology \\ Prószkowska 76, 45-758 Opole, Poland \\ ${ }^{b}$ TurboCare Poland S.A., Powstańców Sląskich 85, 42-701 Lubliniec, Poland
}

\begin{abstract}
Results of scientific research works considering selection of measuring transducer applied for registration of acoustic emission signals generated by on load tap changers are presented in the paper. During the coupling process acoustic emission signals are generated by: the mechanical setup of tap changer, contacts operation, and other phenomena. For most on load tap changers types, the working medium is insulation oil in which the acoustic wave being formed propagates to the steal tank. There exists a possibility to register acoustic emission signals with a piezoelectric transducer attached to the outer tank surface or with a hydrophone immersed in the oil. Acoustic emission signal obtained in such manner contains information which describes operation of the power tap changer and the selector. A comparative analysis of acoustic emission signals generated by on load tap changers working under laboratory conditions, with applied measuring transducers with different transmittances, is presented in the paper. The comparative analysis was performed in order to determine their suitability for on load tap changers technical condition diagnosis. Based on the results achieved, one transducer which allows the registration of acoustic events generated during on load tap changers operation was selected.
\end{abstract}

DOI: $10.12693 /$ APhysPolA.124.395

PACS: 43.40.-r, 43.40.Vn, 43.58.Wc

\section{Introduction}

During its operation, on load tap changer (OLTC) is responsible of fast switching of load currents between appropriate electrical circuits inside the power transformer. This kind of operation, in typical OLTC, causes different physical phenomena occurring during switching process. They are sources of acoustic emission (AE) signals, however, AE from various physical phenomena differ from each other in terms of maximum value and frequency [1-5]. Composition of AE signal highly depends on OLTC design and its actual condition as well as on transducer used to conduct measurements. It is critical for diagnostic purposes that selected transducer should allow to observe as many structures in $\mathrm{AE}$ generated by OLTC operation as possible.

To select transducer most suitable for OLTC diagnostics by AE method a series of measurements was performed. In prepared laboratory setup, group of three different sensors were used to measure AE generated by full scale model of OLTC in different technical conditions. Obtained signals were compared in time and frequency domains.

\section{Transducers used in measurements}

However all tests and measurements presented in this paper were conducted in laboratory conditions, which are

\footnotetext{
*corresponding author; e-mail: a.cichon@po.opole.pl
}

more friendly both to human and equipment, selection of sensors for further use was performed to ensure their capability to operate in harsh industrial environment. Sensors used must be resistant to various chemical agents, like transformer oil, grease, water etc. Furthermore, close proximity of very strong electromagnetic fields requires good electromagnetic shielding. One of important factors, mostly in case of hydrophones, is also allowed operational temperature range - as temperature in OLTC chamber may vary.

According to performed literature survey [6-9], mostly three types of transducers can be used in OLTC AE diagnostics: hydrophones, accelerometers and broadband resonant transducers. Mentioned types of transducers differ from each other in parameters important for accurate $\mathrm{AE}$ signal analysis - frequency band and sensitivity.

Initial research allowed to select three sensors for measurements:

(a) Hydrophone type 8103 by Bruel \& Kjaer,

(b) Accelerometer type $4514 \mathrm{D}$ by Bruel \& Kjaer,

(c) Broadband transducer D9241A by PAC.

Hydrophone type 8103 is small, general purpose sensor with flat frequency response over wide frequency range. It is equipped with double shielded, low noise, integral cable - important in terms of electromagnetic shielding. Its long term allowed temperature range varies from -40 to $+80^{\circ} \mathrm{C}$. Another important feature is high corrosion resistivity which allows its usage in cooling medium of OLTC chamber - transformer oil. Operational fre- 
quency range is between 0.1 and $180 \mathrm{kHz}$ (Fig. 1) and sensitivity around $30 \mu \mathrm{V} / \mathrm{Pa}$.

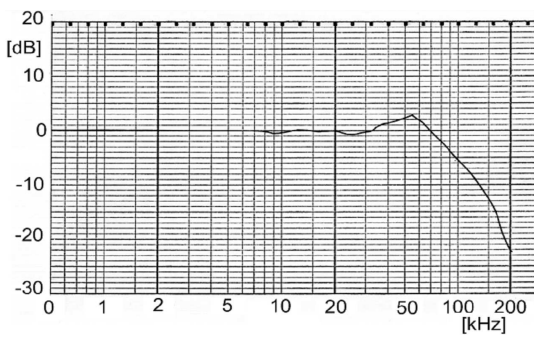

Fig. 1. Frequency characteristic of hydrophone type 8103.

Piezoelectric accelerometer type 4514D is hermetically sealed sensor with insulated base - this is important because of noise reduction during measurements, as sensor is attached to tank of working transformer. It has built-in preamplifier. Typical operational frequency is in $1 \mathrm{~Hz}-$ $10 \mathrm{kHz}$ range (Fig. 2) and sensitivity around $10 \mathrm{mV} / \mathrm{g}$.

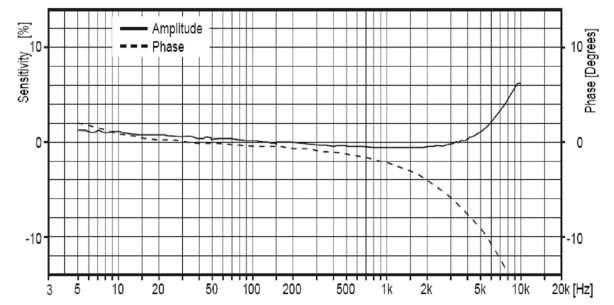

Fig. 2. Frequency characteristic of accelerometer type 4514D.

Acoustic emission sensor D9241A is epoxy sealed, enclosed device designed for measurements in electrically noisy environments. It has ceramic face to ensure proper insulation from transformer tank. Sensor has integral cable with differential BNC connector to help noise reduction. Manufacturer states that typical operational frequency is in $20-180 \mathrm{kHz}$ range (Fig. 3) and sensitivity is around $82 \mathrm{~dB} \mathrm{~V} /(\mathrm{m} / \mathrm{s})$.

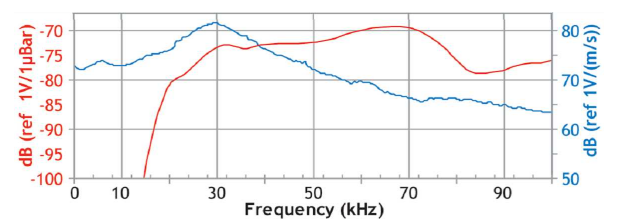

Fig. 3. Frequency characteristic of broadband transducer D9241A.

\section{Laboratory setup}

In order to keep experiment close to reality, but still in laboratory conditions, authors performed measurements on OLTC model which is very similar in construction to OLTC in power transformer. Discussed model consists of securely sealed, rectangular, steel tank of around $1 \mathrm{~m}^{3}$ volume filled with transformer oil. Inside the tank a switching mechanism is placed. The mechanism used in model is separated power switch and selector type VEL 110. Used OLTC switching mechanism is very common in Polish power grid. To decrease height of the switching mechanism, selector has reduced number of taps (six) - this allows smaller tank to be used. Mechanism is driven by electric motor connected mechanically by series of gears and shafts. Model can be operated semi-automatically (button activated relay) or manually (hand driven crank). In performed experiments semi-automatic mode was used as it is very similar to industrial OLTC mode of operation. The only difference is the source of signal initiating switching process (in industrial OLTC signal comes from automatic voltage regulation system, in tested model switching process was initiated by researchers).

Contact transducers were placed close to each other in the middle of model tank wall. To ensure proper contact area between each sensor and tank wall they were attached by specially designed magnetic holders. Hydrophone was immersed inside tank, close to switching mechanism, and fixed to reduce possibility of its movement.

Data collection was performed in parallel. This means that all used transducers required separated channels. This kind of measurement technique was chosen to ensure that all sensors would record the same AE in analyzed model. It is very important, as physical phenomena occurring in OLTC are not always repeatable, even when switching between two, the same, taps multiple times. Each measuring channel consisted of sensor, pre-amplifier, amplifier, band-pass filter and finally - data acquisition computer which was common for all channels. Gains of pre-amplifiers and amplifiers were adjusted individually for each channel to achieve acceptable signal strengths - once set, they were maintained during all the performed measurements. Band-pass filtering was applied in all channels - to limit noises outside of suspected signal band. Used sampling frequency was $500 \mathrm{kHz}$ at all times.

\section{Results of measurements}

Performed series of measurements resulted in obtaining sets of AE signals registered for all sensors and all possible switching sequences. To perform comparative analysis between mentioned sensors, one of the recorded switching sequences was selected for further research. As mentioned before, measurements were performed in parallel, so all sensors were registering the same AE generated by operating OLTC switching mechanism.

AE signal generated by operating OLTC consists of series of acoustic events [10] - as it can be seen on example of signal registered by hydrophone type 8103. Events present in registered $\mathrm{AE}$ are generated by switching sequence taking place during operation of tap selector and 
power switch. Observed sequences lasts around $180 \mathrm{~ms}$ and they can be divided into two parts: from 0 to around $90 \mathrm{~ms}$ and from $90 \mathrm{~ms}$ to $180 \mathrm{~ms}$. First part of signal (0-90 ms) contains AE generated by operating tap selector while the second part holds AE generated by operating power switch. The main difference between both groups of signals is count of individual events taking place in each of them. While operating tap selector generates whole series of signals, power switch is responsible for small number (mostly one) of events. The second difference is in the value of signals which corresponds with its amplitude. In most cases, power switch generates much more powerful acoustic signal, clearly hearable even for human standing next to operating OLTC model. This is because power switch is activated by powerful spring.

As it can be seen in Fig. 4, signal registered by type 8103 hydrophone shows several acoustic events present. They all can be distinguished thanks to three important factors: highly sensitive sensor, relatively small distance from active parts of OLTC, and low signal damping (signal propagation path in oil was mostly unblocked).

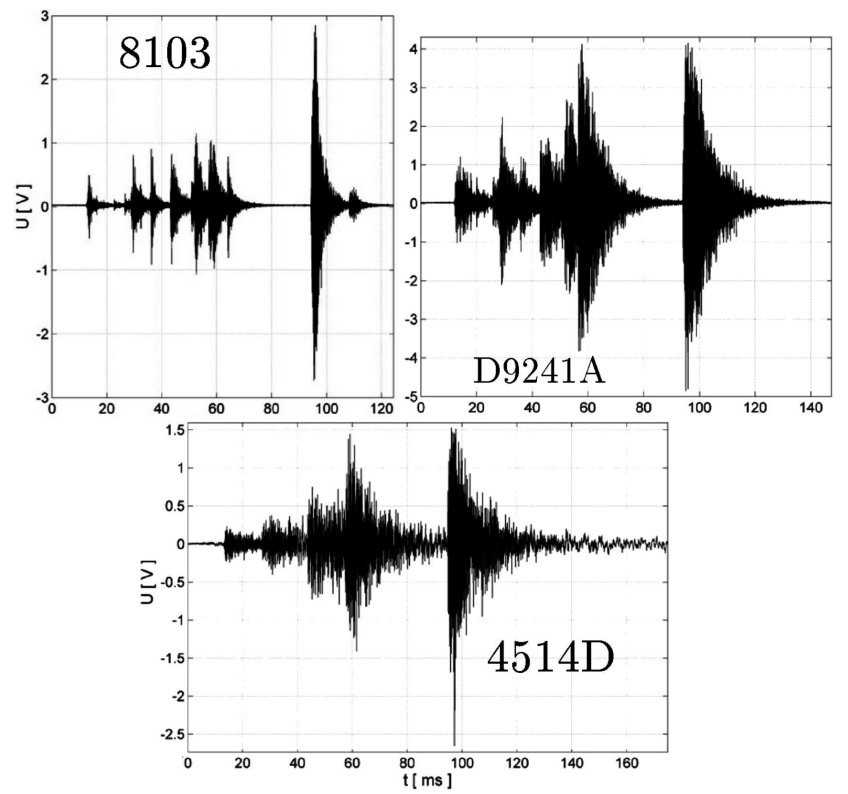

Fig. 4. AE signal registered by hydrophone type 8103 , accelerometer type $4514 \mathrm{D}$, and broadband transducer D9241A.

In case of type $4514 \mathrm{D}$ accelerometer (Fig. 4), which was attached outside the model tank, it is not possible to clearly determine how many individual events are present in registered signal. It appears to be noisy and it is hard to find maximum values of independent $\mathrm{AE}$ events that took place one after another. This is due to narrow frequency band of accelerometer - this means large part of generated signal is not registered at all. In addition, one must take into account influence of steel tank on passing its signals en route from source to sensor.

AE signals registered by broadband transducer D9241A (Fig. 4) are quite similar to those registered by accelerometer, however much better visible and distinguishable. This is due to wider frequency range of D9241A sensor. However, influence of steel tank wall is still visible, because signal is slightly blurred.

Observing signals in time domain provides valuable information regarding registered data. However, in most cases it is worth to transform obtained data and perform extensive analysis in frequency domain [2]. In discussed example, frequency analysis is intended to provide information about power density in frequency spectrum. Most valuable information would be about real sensor operational frequency range and distribution of power density along frequency band with special attention to possible energy concentrations.

As it can be seen in Fig. 5, transformation of AE signal from time to frequency domain provides two-dimensional, time-frequency plot. The third dimension here is the signal power density illustrated by color key showed on each of the spectrograms. Analysis was performed in $0-250 \mathrm{kHz}$ frequency range and in full time spectrum of originally obtained signals $(180 \mathrm{~ms})$.

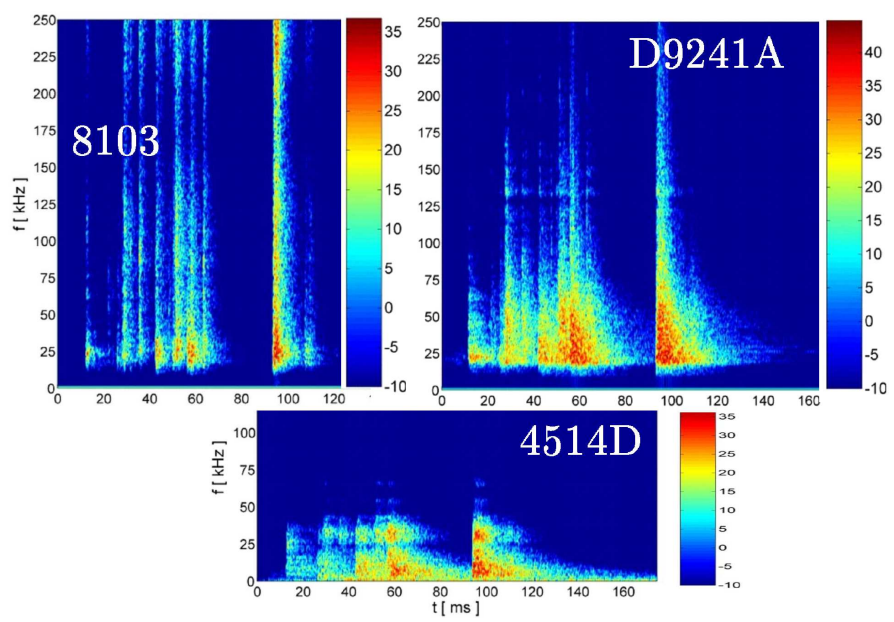

Fig. 5. Spectral power density of signal registered by hydrophone type 8103 , accelerometer type 4514D, and broadband transducer D9241A.

It is clearly visible (Fig. 5) that accelerometer does not register any signals outside $0-50 \mathrm{kHz}$ range. This is of course due to its specific frequency characteristic, however while manufacturer described its operational range between around $1 \mathrm{~Hz}$ and $10 \mathrm{kHz}$, it is evident that sensor picks some signals up to $50 \mathrm{kHz}$. After having a closer look, it is apparent that signals are grouped in two bands: $0-20 \mathrm{kHz}$ (which is dominant) and $20 \mathrm{kHz}$ to $50 \mathrm{kHz}$. Above $50 \mathrm{kHz}$ signal power rapidly decreases. In dominant $0-20 \mathrm{kHz}$ frequency range signal is clearly visible in whole time spectrum with two main power peaks at 60 and $100 \mathrm{~ms}$. It is obvious that this type of diagnostic transducer is suitable only for low frequency AE.

Hydrophone type 8103, as it can be seen in Fig. 5, is able to register signals in $15-250 \mathrm{kHz}$ frequency range. It is largely unclear why this sensor did not picked sig- 
nals in $0.1-15 \mathrm{kHz}$ range (as it should according to its frequency characteristic). Additionally, it appears that it is suitable for measurements above its top rated frequency of $180 \mathrm{kHz}$ as strong signals can be observed up to $250 \mathrm{kHz}$. Unlike the accelerometer, which recorded all very low frequency signals present in almost whole time spectrum (including AE generated by rotating shaft and gears), hydrophone did recorded each of the separate acoustic events so they can be distinguished. Presented spectral power density plot (Fig. 5) has shape of vertical lines, each one representing one acoustic event. As it can be seen, most of the highly powered events are in 20 $50 \mathrm{kHz}$ range. Only the last acoustic event, generated by power switch operation, can be described as highly powerful in almost entire observed frequency spectrum.

While analyzing data regarding broadband transducer D9241A (Fig. 5) one can find out that presented record is in some way similar both to hydrophone and accelerometer spectral power density plots. Broadband transducer did not record almost any signals of frequency below $15 \mathrm{kHz}$ - this is of course due to its frequency characteristic. However, in high frequency band, above 0-90 kHz frequency characteristic provided by sensor manufacturer, signal is still registered but it becomes weak with frequency increase. It can be seen (Fig. 5) that most of the signal power is contained in $20-50 \mathrm{kHz}$ band, around $30 \mathrm{kHz}$ resonant frequency of sensor. Observed structures are slightly blurred (similar to accelerometer), it is believed that this is caused by limited mechanical stiffness of OLTC model steel tank which becomes to vibrate under AE excitation (model tank wall was made of rather thin sheet of metal). It is possible that this feature will not be a problem in full scale measurements, as in real OLTC tank walls are made of much thicker material.

\section{Selecting most suitable sensor}

After a brief presentation of results obtained by performing measurements with each of the sensors (Sect. 4) it becomes evident that each of them has different properties. All tested sensors were initially selected to meet the requirements of measurements conducted in industrial environment. Presented sensors are resistant to conditions that may happen during full scale diagnostic procedures in the field. Judging from performed measurements, acoustic data provided by hydrophone was most suitable for diagnostic use. However, in most occasions, during real industrial measurements access to OLTC tank is restricted, as tank is tightly sealed. This fact limits use of hydrophone to laboratory experiments. The use of accelerometer is much easier in field conditions, as it can be attached to OLTC tank wall. Its main drawback is very narrow frequency characteristic, allowing to observe only signals of low frequencies. On the other hand, broadband sensor is capable to register signals in much wider range - in addition, it can be attached to OLTC tank wall at any time. All in all, after analyzing presented materials, it can be said that most suitable sensor for OLTC, AE diagnostic measurements is broadband transducer (D9241A, in discussed example).

\section{Summary}

This paper presents results of comparative analysis performed to select most suitable AE sensor for industrial OLTC diagnostics measurements. Initial research provided information about typical sensors used worldwide. Three different sensors meeting environmental criteria were selected for further study: hydrophone, accelerometer and broadband frequency transducer. After performing series of measurements in laboratory conditions, close to reality OLTC model, obtained data were analyzed in time and frequency domains. In the end, the broadband transducer was selected as most suitable for performing full scale diagnostic procedures in the field.

\section{Acknowledgments}

The work was financed with the means from the National Research and Development Center within LIDER program.

\section{References}

[1] S. Borucki, Acta Phys. Pol. A 120, 571 (2011).

[2] S. Borucki, T. Boczar, A. Cichoń, Arch. Acoust. 36, 49 (2011).

[3] A. Cichoń, Electrotechn. Rev. 88, 22 (2012) (in Polish).

[4] D. Wotzka, A. Cichoń, T. Boczar, Arch. Acoust. 37, 19 (2012).

[5] A. Cichoń, S. Borucki, D. Wotzka, M. Szmajda, Acta Phys. Pol. A 122, 804 (2012).

[6] A. Cichon, A New Diagnostics Method for Technical Condition of On-Load Tap Changer, Politechnika Opolska, Opole 2011.

[7] M. Foata, R. Beauchemin, C. Rajotte, ESMO, IEEE Proc., Montreal 2000, p. 293.

[8] E. Filho, L.A.L. de Almeida, IMTC, IEEE Proc., Victoria 2008, p. 1051.

[9] D.-J. Kweon, S.-B. Chin, H.-R. Kwak, J.-C. Kim, K.-B. Song, IEEE Trans. Power Deliv. 20, 1976 (2005).

[10] A. Cichoń, P. Frącz, D. Zmarzły, Acta Phys. Pol. A 120, 585 (2011). 